\title{
Epitaxial graphene thermistor for cryogenic temperatures
}

\author{
Sırrı Batuhan Kalkan, Serap Yiğen, Cem Çelebi* \\ Quantum Device Laboratory, Department of Physics, Izmir Institute of Technology, Izmir 35430, Turkey
}

\section{A R T I C L E I N F O}

\section{Article history:}

Received 17 May 2018

Received in revised form 10 July 2018

Accepted 13 July 2018

Available online 18 July 2018

\section{Keywords:}

Epitaxial graphene

Encapsulation

NTC thermistor

Low-temperature

\begin{abstract}
A B S T R A C T
The thermal responsivity of monolayer epitaxial graphene grown on the Si-face surface of semi-insulating $\mathrm{SiC}$ substrate is investigated as a function of temperature below $300 \mathrm{~K}$. The measurements showed that adsorption/desorption of atmospheric adsorbates can randomly modify the electrical characteristics of graphene which is indeed undesirable for consistent temperature sensing operations. Therefore, in order to avoid the interaction between graphene layer and adsorbates, the grown graphene layer is encapsulated with a thin $\mathrm{SiO}_{2}$ film deposited by Pulsed Electron Deposition technique. Temperature dependent resistance measurement of encapsulated graphene exhibited a clear thermistor type behavior with negative temperature coefficient resistance character. Both the sensitivity and transient thermal responsivity of the $\mathrm{SiO}_{2}$ /graphene/SiC sample were found to be enhanced greatly especially for the temperatures lower than $225 \mathrm{~K}$. The experimentally obtained results suggest that $\mathrm{SiO}_{2}$ encapsulated epitaxial graphene on SiC can be used readily as an energy efficient and stable temperature sensing element in cryogenic applications.
\end{abstract}

(c) 2018 Elsevier B.V. All rights reserved.

\section{Introduction}

Graphene has been considered to be a promising 2D material for the realization of new generation microelectronic and optoelectronic devices with planar architecture owing to its high charge carrier mobility [1], large surface-to-volume ratio [2] and excellent optical transparency [3]. Besides, superior thermal conductivity (TC) of graphene, reaching a value of about $5300 \mathrm{~W} / \mathrm{m} . \mathrm{K}[4,5]$ at room temperature, has motivated researchers to explore the potential use of this 2D material also in temperature sensor technology. The 2D planar structure allows graphene to be directly implanted to integrated circuits as a functional temperature sensing element. However, the restrictions due to sophisticated lithography techniques [6], subsequently low temperature sensitivity and slow response speed of bare graphene [7-9] are the main constrains for the development of graphene based thermal sensors.

The transfer process and substrate choice play an essential role for graphene based temperature sensors. The defects and residues created during the transfer process for chemical vapor deposited (CVD) graphene and as well as relatively low TC of underlying substrates [10] such as silicon dioxide $\left(\mathrm{SiO}_{2}\right)(1.3 \mathrm{~W} / \mathrm{m} . \mathrm{K})$ [11] and silicon nitride $(\mathrm{SiN})(4 \mathrm{~W} / \mathrm{m} . \mathrm{K})$ [12], greatly hinder the transient thermal responsivity of graphene layer. In contrast to CVD

\footnotetext{
* Corresponding author.

E-mail address: cemcelebi@iyte.edu.tr (C. Çelebi).
}

graphene, epitaxial graphene (EG) is ease of production on silicon carbide $(\mathrm{SiC})$, which plays into our hands with a large TC of around $300 \mathrm{~W} / \mathrm{m} . \mathrm{K}$ [13] when compared to these low TC materials as a substrate: Silicon atoms evaporate from SiC surface at high temperatures and carbon atoms that are left on the surface forms the graphene layer. Therefore, single atom thick EG can be grown readily on SiC's polar surfaces (Si-face and C-face) without need of any transfer and/or sophisticated deposition processes like CVD and sputtering that are the most commonly used techniques to produce metal, semiconductor or ceramic based conventional thermistor devices.

Previous studies showed that extremely large surface-tovolume ratio of graphene leads to undesirable electrical instabilities when it is integrated as a functional component in electronic or optoelectronic devices operating under atmospheric conditions [14]. The adsorbates like $\mathrm{O}_{2}$ and $\mathrm{H}_{2} \mathrm{O}$ in air serve as surface trap states and sequential adsorption/desorption of these molecules drastically alter the electronic transport characteristics of graphene via charge transfer doping phenomenon. In the case of bare graphene exposed to these atmospheric gases, heating and/or cooling process is expected to promote further the adsorption/desorption of these above mentioned adsorbates which may cause abrupt variations both in the electrical and thermal conductivities of graphene $[15,16]$. Thus, the conductivity of graphene is strongly influenced by the atmospheric gases and humidity fluctuations as it should depend only on temperature variations. This causes different conductivity values for the same temperature, 
which must be avoided. Therefore, for stable and reliable temperature sensing operations, the interaction of graphene with the surrounding adsorbates should be necessarily prevented, for example, by encapsulating or passivating the graphene layer with an appropriate material. However, all the graphene based thermistor type sensors investigated in earlier studies do not comprise any encapsulation protection [6-9].

In this work, we report the thermal response of monolayer EG that was grown on a single crystal semi-insulating (SI) SiC substrate by high temperature vacuum annealing process. In order to avoid adsorbate induced possible variations in the charge carrier dynamics of graphene, the adsorbates already stuck on bare epitaxial graphene (BEG) in air were removed by UV light exposure under vacuum conditions. The UV exposure process is necessary to ensure stable and repeatable temperature sensing operations that relies only on the intrinsic thermal and electrical characteristics of the graphene layer itself. Adsorbate free graphene layer were encapsulated with a thin $\mathrm{SiO}_{2}$ film deposited by state-ofthe-art Pulsed Electron Deposition (PED) technique. The resistance vs temperature (R-T) measurements showed that $\mathrm{SiO}_{2}$ encapsulated epitaxial graphene (EEG) is a typical negative temperature coefficient (NTC) material which exhibits rapid electrical resistance increase with temperature decrease. We have also found that the encapsulation of graphene yields reversible thermal sensing operations unlike BEG samples. Transient thermal responsivity measurements implied that $\mathrm{SiO}_{2}$ encapsulated EG on $\mathrm{SiC}$ can be used readily as a temperature sensing element especially for low temperature applications.

\section{Experimental}

Monolayer epitaxial graphene is grown on the Si terminated face of a ( $4 \mathrm{~mm} \times 10 \mathrm{~mm}) \mathrm{SI} 4 \mathrm{H}-\mathrm{SiC}$ substrate annealed at an elevated temperature of $1450^{\circ} \mathrm{C}$ for $20 \mathrm{~min}$ in a UHV chamber $\left(\sim 2 \times 10^{-10}\right.$ mbar). Prior to the graphene growth, SiC substrate degassed at $600^{\circ} \mathrm{C}$ over night and then the temperature is raised to $1100^{\circ} \mathrm{C}$ for about $10 \mathrm{~min}$ to remove the native oxide layer. During the growth experiment, the temperature of the sample was measured remotely by using an optical pyrometer with $\pm 1^{\circ} \mathrm{C}$ resolution. At the growth temperature of $1450^{\circ} \mathrm{C}$, the Si atoms sublimate from $\mathrm{SiC}$ surface into vacuum and left behind $C$ atoms forms the graphene layer by mimicking the hexagonal lattice structure of underlying $\mathrm{SiC}$ crystal. To verify the growth of epitaxial graphene and as well as to determine its thickness, we conducted single point Raman spectroscopy measurements by a green laser with $532 \mathrm{~nm}$ excitation wavelength. The details of graphene growth experiments and Raman spectroscopy analysis can be found in our previous studies $[17,18]$.

The morphology of grown graphene layer was characterized by tapping mode Atomic Force Microscopy (AFM) topography measurements. The AFM image of BEG layer (Fig. 1(a)) exhibits typical $\mathrm{SiC}$ background based large terraces and graphene related flaky structures appearing with blurry wrinkles lying on these parallel terraces. Small amount of local and darker regions correspond to a few layer BEG flakes. Following the structural and morphological analysis of epitaxial graphene, $\mathrm{Cr} / \mathrm{Au}(3 \mathrm{~nm} / 80 \mathrm{~nm}$ ) interconnect source/drain pads were deposited thermally on the two sides of the grown layer for conducting two-terminal I-V measurements (see Fig. 1(b)). To promote desorption of adsorbates which were already stuck on graphene in air, the sample was exposed to UV light for a period of $3 \mathrm{~h}$ under high vacuum conditions $\left(10^{-5} \mathrm{mbar}\right)$. The UV irradiation wavelength is specifically selected to be $254 \mathrm{~nm}$ since it is energetically sufficient enough to remove the $\mathrm{O}_{2}$ and $\mathrm{H}_{2} \mathrm{O}$ molecules adsorbed by the graphene layer.
After the adsorbate removal process, the sample surface was encapsulated with a $100 \mathrm{~nm}$ thick $\mathrm{SiO}_{2}$ layer by Neocera PEBS32 PED system. The R-T measurements were conducted using $5 \mathrm{~K}-300 \mathrm{~K}$ Janis cold head optical cryostat equipped with a Pfeiffer Hi-Cube pump station. Lakeshore 331 temperature controller and a calibrated reference thermocouple were used for temperature control and measurements, respectively. In order to ensure good thermal contact and electrical insulation at the same time, low temperature compatible ceramic epoxy was employed for bonding the sample on a Copper (Cu) plate. The $\mathrm{Cu}$ plate with the sample on top were mounted on the sample holder of cold head (Fig. 1(d)). The electrical characterizations of the sample were done by using Keithley 2400 Source Meter and Keithley 6220 Picoammeter. The R$T$ measurements of the sample were conducted under a bias voltage of $\mathrm{V}_{\mathrm{b}}=2 \mathrm{~V}$.

\section{Results and discussion}

The I-V characteristics of BEG sample obtained before and after in-vacuum UV light treatment were plotted in Fig. 2(a) The measurements show that room temperature resistance $\left(R_{300 \mathrm{~K}}\right)$ of as-grown BEG sample is decreased from $\sim 2.3 \mathrm{k} \Omega$ to $\sim 1.2 \mathrm{k} \Omega$ after $3 \mathrm{~h}$ of UV light exposure. The reduction in the resistance of graphene can be explained as in the following manner. Epitaxial graphene grown on the Si-terminated face of $\mathrm{SiC}$ is known to be intrinsically n-type doped due to charge transfer between graphene layer and $\mathrm{SiC}$ substrate underneath [19]. The atmospheric molecules such as $\mathrm{O}_{2}$ and $\mathrm{H}_{2} \mathrm{O}$ are adsorbed readily by the graphene layer when it is exposed to air. These adsorbates behave like electron trapping surface states and thus reduce the electron density of n-type epitaxial graphene as schematically illustrated in Fig. 2(b). As the UV light promotes desorption of these adsorbates from the sample surface into vacuum, the trapped electrons are released back to the graphene layer via charge transfer doping mechanism. Therefore, the $R_{300 K}$ of adsorbate free BEG becomes lower than that of as-grown BEG measured prior to the UV light exposure process. The R-T characteristics of BEG sample measured before and after UV exposure were compared in Fig. 2(c) and (d). The resistance of as-grown BEG with adsorbates increases almost like exponentially as a function of decreasing temperature (Fig. 2(c)) and resembles to that of a typical thermistor element with NTC character. The overall resistance variation for as-grown BEG was determined to be about $21 \%$ for the temperature range between $25 \mathrm{~K}$ and $300 \mathrm{~K}$. After the adsorbate removal process with UV light, the R-T characteristics of BEG becomes similar to that of a conventional metal at high temperatures whereas exhibits an upturn at around $150 \mathrm{~K}$ (Fig. 2(d)). This peculiar temperature dependent resistance behavior of epitaxial graphene on $\mathrm{SiC}$ has been previously attributed to the weak-localization and electron-electron interaction phenomena [20]. It is clearly seen that adsorbate free graphene cannot be used as a thermistor due to the continuous interaction of ambient gases with the graphene layer. The resistance characteristics of bare graphene sensor strongly depends on the partial pressure of the surrounding adsorbates. These random interactions are undesirable for ultimate electrical stability. Our experiments showed that the resistance of graphene can change simultaneously with the adsorption/desorption of electron trapper adsorbates. Thus, the resistance of our fabricated sensor at $200 \mathrm{~K}$ becomes the same as the one measured at $130 \mathrm{~K}$ due to the parabolic behavior of the R-T curve after UV light illumination (Fig. 2(d)). Therefore, it is of crucial importance to encapsulate graphene in order to stabilize and rectify its temperature sensing capability.

Following the R-T measurements of BEG conducted before and after UV light illumination, the sample was encapsulated with a $100 \mathrm{~nm}$ thick $\mathrm{SiO}_{2}$ layer by using PED technique. For comparison, 


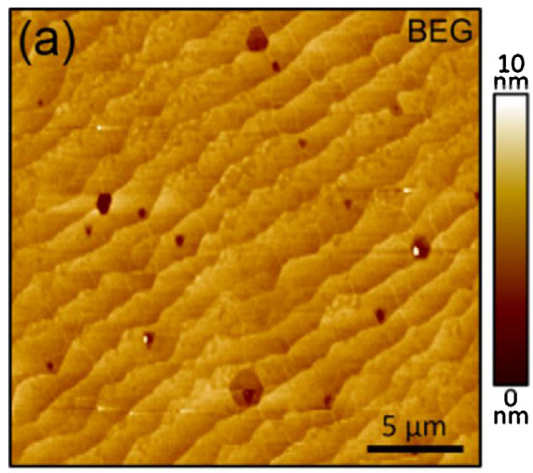

(b)
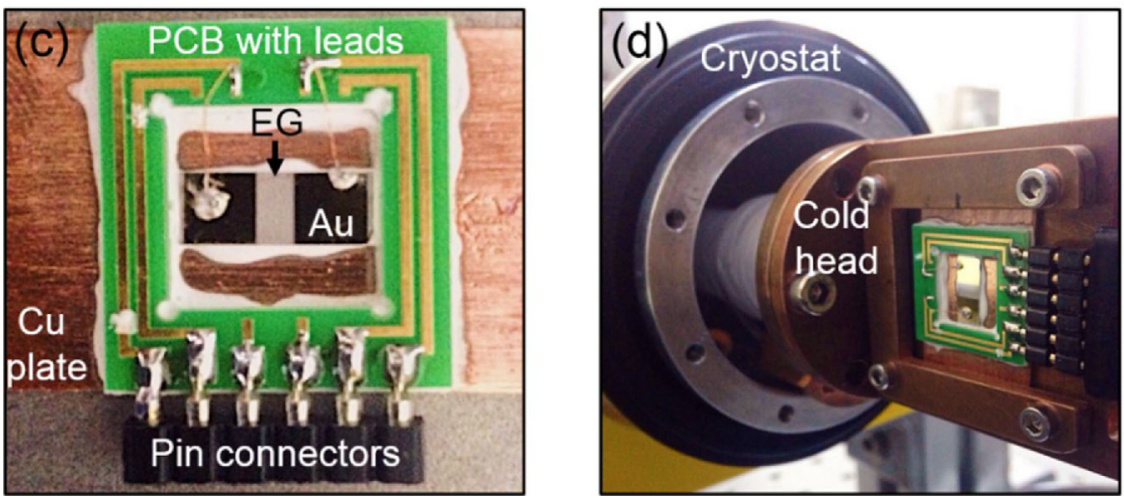

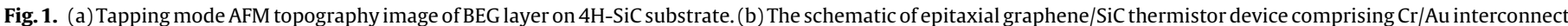

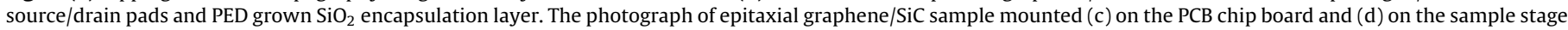
of $5 \mathrm{~K}-300 \mathrm{~K}$ Janis cryostat.

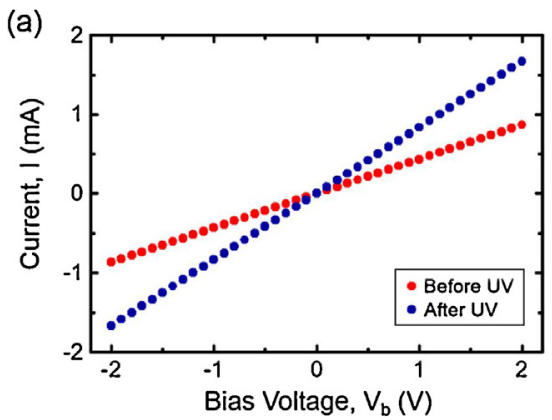

(b)
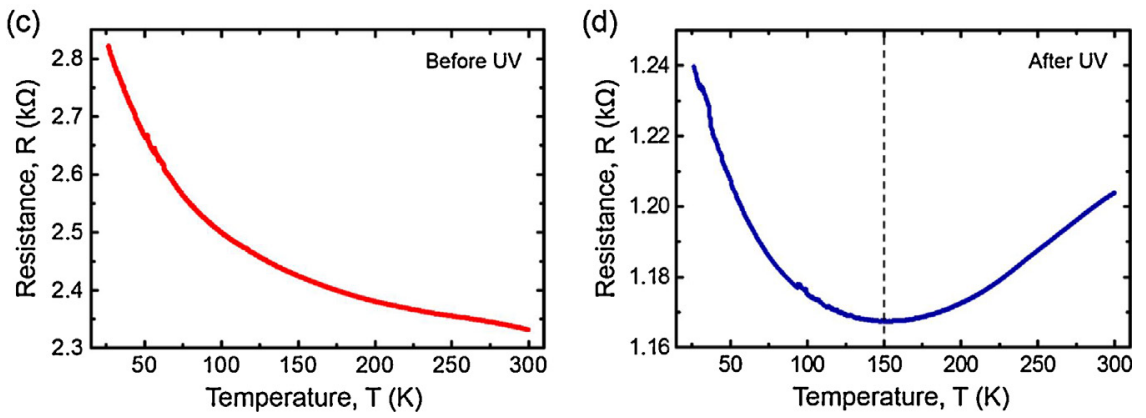

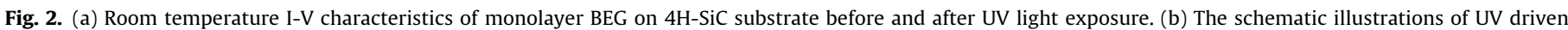

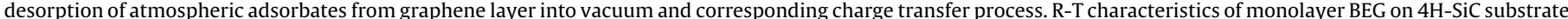
(c) before and (d) after UV light illumination.

the resistance of EEG sample was also measured as a function of temperature between $25 \mathrm{~K}$ and $300 \mathrm{~K}$. Fig. 3(a) displays the R-T plot of EEG sample, which provides a direct view for the asymptotic increase of resistance upon cooling. The observed trend in $\mathrm{R}$-T measurement is a clear indicator of a typical thermistor behavior with NTC resistance character. The overall change in resistance was determined to be about $7.5 \times 10^{3} \%$ which is greater than the total resistance variation of as-grown BEG sample (21\%) measured before the UV light exposure. It should be noted that, in contrary to as-grown BEG sample, we observed no deviation in the R-T characteristics of EEG before and after UV light illumination, as well as in cool-down and warm-up cycles. After $\mathrm{SiO}_{2}$ encapsulation, the 
(a)

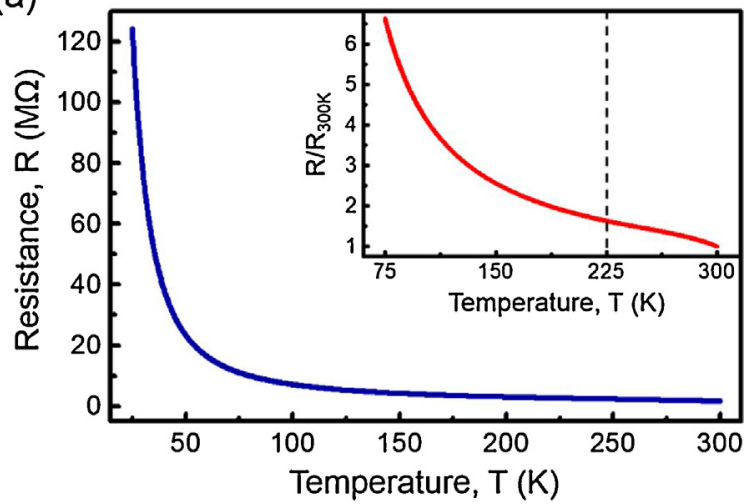

(b)

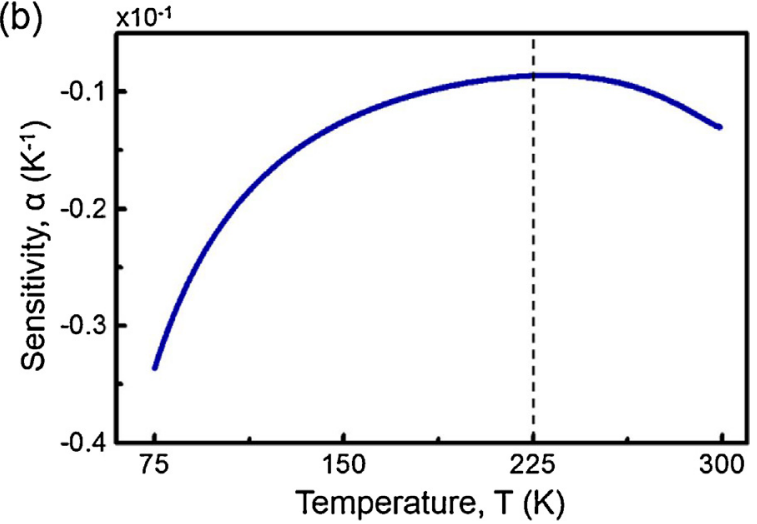

Fig. 3. (a) R-T characteristics of EEG on $4 \mathrm{H}-\mathrm{SiC}$ substrate. Inset displays the change in normalized resistance $\left(R / R_{300 K}\right)$ of EEG sample measured in a temperature range between $75 \mathrm{~K}$ and $300 \mathrm{~K}$. (b) Thermal sensitivity curve of EEG sample determined in a temperature range between $75 \mathrm{~K}$ and $300 \mathrm{~K}$.

$\mathrm{R}_{300 \mathrm{~K}}$ of the sample rises to such a value as high as $1.6 \mathrm{M} \Omega$. This high resistance and NTC behavior suggest that EG layer is converted from a conductor into an insulator or even a semiconductor. In the case of plasma involved thin film deposition processes, such as PED, low energy plasma exposure on graphene results in an increment around one order of magnitude in the $R_{300 K}$ value [19]. However, the $R_{300 K}$ of our EEG was found to be about $7 \times 10^{2}$ times greater than that of BEG measured before the $\mathrm{SiO}_{2}$ deposition process. Such a large increment in the resistance can also be associated with the charge trap states formed at the $\mathrm{SiO}_{2}$ /graphene interface.

As one of the most important characteristics of a thermistor device, the normalized resistance $\left(R / R_{300 K}\right)$ is used to determine the nominal resistance value at a specified temperature. The $R / R_{300 K}$ of EEG sample was plotted at the inset of Fig. 3(a) as a function of temperature ranging between $75 \mathrm{~K}$ and $300 \mathrm{~K}$. For $75 \mathrm{~K}$, the $\mathrm{R} / \mathrm{R}_{300 \mathrm{~K}}$ of the sample reaches to a value of around 6.5 and tends to grow abruptly for lower temperatures consistent with the corresponding R-T measurement shown in Fig. 3(a). The thermal sensitivity $(\alpha)$ of the fabricated EEG sample is extracted from the relation $\alpha=$ $-\beta / T^{2}$, where $\beta$ is the empirical calibration constant which scales the response of resistance to temperature change for a NTC thermistor. The $\beta$-factor in the sensitivity relation is determined by using the following expression,

$\frac{R}{R_{300 K}}=\exp \left\{\beta\left(\frac{1}{T}-\frac{1}{300}\right)\right\}$

Below $225 \mathrm{~K}$, where the sensitivity is larger and NTC characteristics becomes more pronounced, the $\beta$-factor of the sample was determined to be about 235. As seen in Fig. 3(b), the thermal sensitivity of EEG sample decreases (increases) above (below) $225 \mathrm{~K}$ as a function of decreasing temperature. These two distinct sensitiv- ity behaviors are correlated with two different R-T characteristics observed above and below $225 \mathrm{~K}$. As seen at the inset of Fig. 3(a), the variation in the resistance above $225 \mathrm{~K}$ is slightly different from the one measured below $225 \mathrm{~K}$. This observed phenomenon can be explained in terms of the interplay between electron trapping mechanism at $\mathrm{SiO}_{2}$ /graphene interface and charge transfer process from $\mathrm{SiC}$ to graphene occurring at high temperatures. The defects created during the deposition of $\mathrm{SiO}_{2}$ dielectric layer on graphene give rise to charge trap states at $\mathrm{SiO}_{2}$ /graphene interface. The electron density in graphene is considerably lowered due to the trapping of electrons by these interface states. Since the electron density is reduced, the Fermi level of BEG shifts towards a lower energy state close to the Dirac point leading to a large increment in the $R_{300 \mathrm{~K}}$ value of the sample after the encapsulating process. It is known that a Schottky junction (with a barrier height of $\sim 1 \mathrm{eV}$ ) is formed at the EG/SiC interface [21]. The reduction of Fermi level also lowers the Schottky barrier height and hence the thermally excited electrons in the depletion layer of SiC are readily transferred to graphene via thermionic emission and/or tunneling phenomenon at high temperatures. This process partially increases the electron density and thus contributes to the electrical conductivity of graphene layer. However, as the temperature is decreased down from $300 \mathrm{~K}$, the number of thermal electrons is reduced in the depletion layer of SiC. Therefore, the amount of electrons transferred from $\mathrm{SiC}$ to graphene is decreased accordingly. The competition between the injection (from $\mathrm{SiC}$ ) and trapping (by $\mathrm{SiO}_{2}$ ) of electrons in through graphene layer dominates the $\mathrm{R}-\mathrm{T}$ characteristics and leads to a substantial decay in the thermal sensitivity of EEG sample as the temperature decreased from $300 \mathrm{~K}$ to $225 \mathrm{~K}$ (Fig. 3(b)). The experimentally obtained sensitivity data imply that the contribution of thermal electrons from $\mathrm{SiC}$ to graphene is greatly suppressed around $225 \mathrm{~K}$. The increment of resistance and thus the enhancement of thermal sensitivity at lower temperatures below $225 \mathrm{~K}$ can be related to the electron-electron interaction which is strongly sensitive to disorders in graphene layer [20]. For high (300 K) and low (75 K) temperature values, the average variations in the thermal sensitivity of the fabricated $\mathrm{SiO}_{2} / \mathrm{EG} / \mathrm{SiC}$ thermistor devices were found to be $5.6 \%$ and $2.1 \%$, respectively.

The transient thermal responsivity of EEG sample was determined relative to that of a reference thermocouple mounted on the sample stage of our cryostat system (Fig. 4). When the temperature was set to a certain value like $\mathrm{T}_{0}$ by the temperature controller, the heater of cryostat is periodically switched on and off in order to bring the temperature to its set value. During this operation, the measured temperature oscillates with small amplitudes like $\Delta \mathrm{T}$ in a period defined only by the on and off states of the heater. The thermal responsivity of EEG sample was characterized with respect to these small temperature oscillations that occur around different cryostat temperatures set to $300 \mathrm{~K}, 225 \mathrm{~K}, 150 \mathrm{~K}$ and $75 \mathrm{~K}$. Fig. 4 shows the resistance variations in EEG sample $\left(\Delta R / R_{0}\right)$ due to the temperature alterations $\left(\Delta \mathrm{T} / \mathrm{T}_{0}\right)$ read by the reference thermocouple. The peak positions of resistance and temperature variations were compared with each other in order to determine the relative thermal response time $\left(\Delta t=t_{T c}-t_{E E G}\right)$, where $t_{T c}$ and $t_{\mathrm{EEG}}$ are the peak position of the reference thermocouple and EEG sample, respectively. For a clear comparison, the peak position of reference thermocouple $\mathrm{t}_{\mathrm{Tc}}$ were indicated by dashed lines in Fig. 4. Except for the set temperature $T_{0}=225 \mathrm{~K}$, where the thermal sensitivity of EEG sample is around minimum (Fig. 3(b)), the peak to peak value of the resistance variation $\left(\Delta R / R_{0}\right)_{p p}$ is higher than that of the corresponding temperature change $\left(\Delta \mathrm{T} / \mathrm{T}_{0}\right)_{\mathrm{pp}}$. For high temperatures close to $300 \mathrm{~K}$, the EEG sample has a low response speed with a measurement delay of about $\Delta t=23 \mathrm{~s}$ relative to the $t_{\mathrm{Tc}}$ of reference thermocouple. However, we found that the $\Delta t$ value of EEG sample decreases and thus the response speed increases as the 
(a)

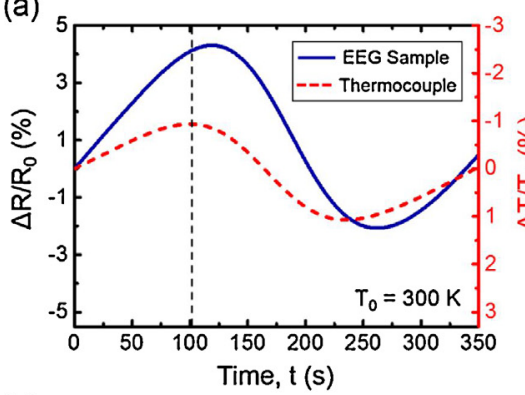

(c)

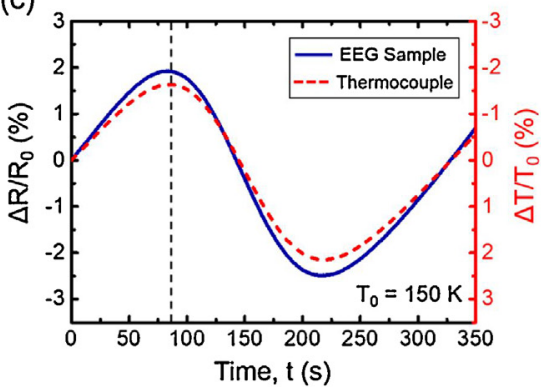

(b)

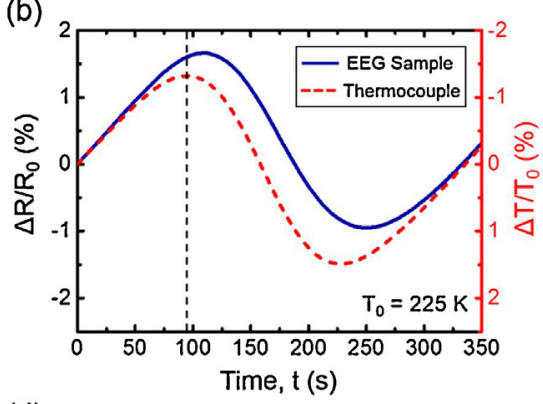

(d)

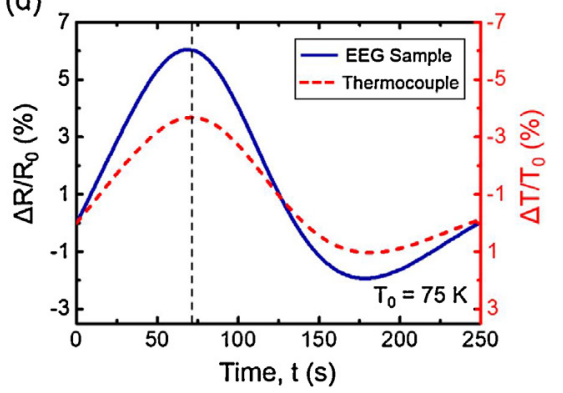

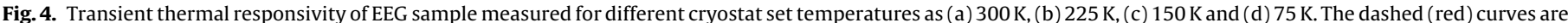

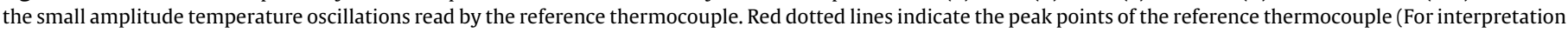
of the references to colour in this figure legend, the reader is referred to the web version of this article).

temperature is reduced well below $300 \mathrm{~K}$. At around $\mathrm{T}_{0}=150 \mathrm{~K}$, the response time of EEG sample with $\Delta \mathrm{t}=-2 \mathrm{~s}$ greatly matches to that of the reference thermocouple. Further measurements show that the response speed of EEG sample is higher than the response speed of reference thermocouple especially for the temperatures below $150 \mathrm{~K}$.

\section{Conclusion}

The thermal response of monolayer epitaxial graphene grown on $\mathrm{SiC}$ was investigated at low temperatures ranging between $25 \mathrm{~K}$ and $300 \mathrm{~K}$. The measurements showed that the R-T characteristics of bare graphene is very sensitive to adsorption/desorption of atmospheric adsorbates which greatly modify the charge carrier dynamics of graphene. In order to prevent the undesirable interaction of adsorbates with epitaxial graphene, the samples were encapsulated with a thin $\mathrm{SiO}_{2}$ dielectric film deposited by PED technique. We found that the encapsulation process increased the resistance of graphene by a factor of about $7 \times 10^{2}$ due to the combination of plasma involved deposition process and charge trap states formed at the interface between $\mathrm{SiO}_{2}$ encapsulation layer and epitaxial graphene. The R-T measurements of encapsulated epitaxial graphene exhibited a clear thermistor behavior with negative temperature coefficient resistance character. The measurements, conducted relative to a calibrated reference thermocouple, showed that the thermal sensitivity and responsivity of encapsulated epitaxial graphene are greatly enhanced for the temperatures lower than $225 \mathrm{~K}$. The reduced power output, achieved after $\mathrm{SiO}_{2}$ encapsulation process, can prevent the self-heating of the sample and therefore enables one to utilize epitaxial graphene in low temperature sensing applications.

\section{Acknowledgement}

The authors would like to thank Dilce Özkendir for her assistance and help during the graphene growth experiments.

\section{References}

[1] K.I. Bolotin, K.J. Sikes, Z.D. Jiang, M. Klima, G. Fudenberg, J. Hone, P.H. Kim, H.L. Stormer, Solid State Commun. 146 (2008) 351.

[2] F. Schedin, A. Geim, S. Morozov, E. Hill, P. Blake, M. Katsnelson, K. Novoselov, Nat. Mater. 6 (2007) 652.

[3] F. Bonaccorso, Z. Sun, T.A. Hasan, A.C. Ferrari, Nat. Photonics 4 (2010) 611.

[4] A.A. Balandin, S. Ghosh, W. Bao, I. Calizo, D. Teweldebrhan, F. Miao, C.N. Lau, Nano Lett. 8 (2008) 902.

[5] E. Pop, V. Varshney, A.K. Roy,MRS Bull. 37 (2012), 12731281.

[6] H. Al-Mumen, F. Rao, L. Dong, W. Li, the 8th Annual IEEE International Conference on Nano/Micro Engineered and Molecular Systems, 2013, pp. 1135-1138.

[7] E.I. Ionete, B.F. Monea, M. Vijulie, A. Soare, S.M. Iordache, A.M. Iordache, I. Stamatin, A. Salceanu, 2014 International Conference and Exposition on Electrical and Power Engineering (EPE), 2014, pp. 774-777.

[8] R. Pawlak, M. Lebioda, J. Rymaszewski, W. Szymanski, L. Kolodziejczyk, P. Kula, Sensors 17 (2017) 51

[9] P. Sahatiya, S.K. Puttapati, V.V.S.S. Srikanth, S. Badhulika,Flex. Print. Electron. 1 (2016), 025006.

[10] B. Davaji, H.D. Cho, M. Malakoutian, J.-K. Lee, G. Panin, T.W. Kang, C.H. Lee, Sci. Rep. 7 (2017) 8811.

[11] M.G. Burzo, P.L. Komarov, P.E. Raad, IEEE Trans. Compon. Packag. Technol. 26 (2003) 80 .

[12] H. Ftouni, C. Blanc, D. Tainoff, A.D. Fefferman, M. Defoort, K.J. Lulla, J. Richard, E. Collin, O. Bourgeois,Phys. Rev. B 92 (2015), 125439.

[13] R. Wei, S. Song, K. Yang, Y. Cui, Y. Peng, X. Chen, X. Hu, X. Xu,J. Appl. Phys. 113 (2013), 053503.

[14] S.B. Kalkan, H. Aydın, D. Özkendir, C. Çelebi,Appl. Phys. Lett. 112 (2018), 013103.

[15] D. Kong, L.T. Le, Y. Li, J.L. Zunino, W. Lee, Langmuir 28 (2012) 13467

[16] C. Yan, J. Wang, P.S. Lee, ACS Nano 9 (2015) 2130.

[17] E. Kuşdemir, D. Özkendir, V. Fırat, C. Çelebi,J. Phys. D: Appl. Phys. 48 (2015), 095104.

[18] C. Çelebi, C. Yanık, A.G. Demirkol, İsmet İ. Kaya, Appl. Surf. Sci. 264 (2013) 56.

[19] S. Kopylov, A. Tzalenchuk, S. Kubatkin, V.I. Falko,Appl. Phys. Lett. 97 (2010), 112109.

[20] J.C. Prestigiacomo, A. Nath, M.S. Osofsky, S.C. Hernndez, V.D. Wheeler, S.G. Walton, D.K. Gaskill, Sci. Rep. 7 (2017) 41713

[21] S. Shivaraman, L.H. Herman, F. Rana, J. Park, M.G. Spencer,Appl. Phys. Lett. 100 (2012), 183112.

\section{Biographies}

Sirrı Batuhan Kalkan Mr. Sırrı Batuhan Kalkan obtained his BSc. degree in 2015 from the Physics Department of İstanbul University. He has recently completed his Master's degree in the Physics Department of Izmir Institute of Technology (IZTECH). 
Serap Yiğen Dr. Serap Yiğen obtained her BSc. degree in 2012 from the Physics Department of IZTECH. She got her Ph.D. in 2017 from Physics Department at Concordia University, Canada. She is currently working as a Postdoctoral researcher in the Quantum Device Laboratory of the Physics Department at IZTECH.
Cem Celebi Dr. Cem Celebi obtained his Ph.D. in 2009 from Applied Physics Department of Eindhoven University of Technology in the Netherlands. He is working as Associate Professor in the Physics Department of IZTECH. He is the principal investigator and group leader of Quantum Device Laboratory at IZTECH. For further information: http://qdl.iyte.edu.tr/ 